\title{
Optical Gap Measurements of Boron Nitride Nanotubes by EELS
}

\author{
R. Arenal *, O. Stephan**, M. Kociak**, D. Taverna**, A. Loiseau***, C. Colliex** \\ * EMC group, MSD Argonne National Laboratory, IL 60439 Argonne - USA \\ ** LPS, Université Paris-Sud and CNRS, 91405 Orsay - France \\ *** LEM, UMR 104 CNRS-ONERA, 92322 Châtillon - France
}

Boron nitride nanotubes (BNNTs) have attracted considerable interest due to their unique physical properties, which make them a possible alternative to their carbon brethren in regards to possible applications. Nevertheless, in contrast to CNTs for which many studies have been focused in their electronic and optical properties, there are almost no data for BNNTs [1]. The study of the low energy-loss (less than $50 \mathrm{eV}$ ) provides direct access to the dielectric properties of a material. In this contribution we present spatially resolved low-loss EELS measurements on individual BNNTs. These experiments represent the first measurement of the optical gap of a material (in this case individual BNNTs) using EELS aloof spectroscopy.

The samples of BNNTs used in this work were synthesized using laser vaporization technique, described in $[2,3]$, and they contain high quantities of single-walled (SW)-BNNTs. Experiments were performed using the Orsay VG HB501 STEM dedicated EELS microscope operating at $100 \mathrm{kV}$. This kind of study on individual NTs requires a high spatial (probe $<0.5 \mathrm{~nm}$ ) and energetic (close to $0.2 \mathrm{eV}$ ) resolutions. These conditions are provided by the STEM in combination with a highly efficient 2D detector. The spectra were recorded employing two kinds of acquisition modes. One was the spectrum-line mode: 40-60 spectra were acquired for each probe position following a line across the tube. In the second one the beam was rastered on a nanoarea centered on the nanotube. This acquisition mode improves noticeably the signal to noise ratio. The average acquisition time was $120 \mathrm{~ms}$ for each spectrum. In order to disentangle the inelastic signal at low energy from the tail of the zero-loss peak (ZLP), a deconvolution procedure based on a Richardson-Lucy algorithm [4] was applied in combination with a subtraction operation of the ZLP. Previously the spectra were realigned and summed. All these results were interpreted using the classical continuum dielectric theory [5].

In this work we investigated more than $50 \mathrm{NTs}$, including individual SW- and MW-BNNTs, as well as ropes of BNNTs. Fig. 1 (a) shows bright field images of a SW and a triple-walled (TW) NTs, respectively. Fig. 1 (b) displays three EEL spectra recorded on these tubes. The SW's spectrum (i) was acquired in the rastered mode. The spectra corresponding to the TW-NT were selected from a spectrum-line for two different probe positions: the spectrum (ii) was acquired with an impact parameter (distance between the object and the electron beam) of about $2 \mathrm{~nm}$ and the other one (iii) was recorded on the TW-NT (penetrating geometry). All the three spectra display two groups of modes, a first one in the 6-9 eV range and a second one centered at 16 $\mathrm{eV}$. The main differences between the spectra (i) and (ii) and spectrum (iii) lie in a higher intensity in the second mode of the doublet at the onset of the spectrum and a small extra intensity above $20 \mathrm{eV}$, which come from extra contributions of volume modes excited in these objects for a penetrating geometry. Fig 1 (c) shows the imaginary part of the dielectric constant of $\mathrm{h}-\mathrm{BN}$ for an orientation where the momentum transfer is perpendicular to the c-axis $(\operatorname{Im}(\varepsilon \perp))$. This was obtained after a Kramers-Kronig analysis of an EEL spectrum acquired in a highly purity thin foil of h-BN. This result is in agreement with the experimental dielectric constants published in [6]. We note the high similarity between the spectra corresponding to the NTs and this curve. This suggests that the different modes of the NTs' spectra can mainly be attributed to the $\operatorname{Im}(\varepsilon \perp)$ contribution in the dielectric response of the tubes. Fig. 2 displays a series of deconvoluted spectra from the vacuum to a SW-BNNT. Such spectra contain the signature of surface modes excited in a near-field geometry 
where the electron beam is focused at an aloof geometry of the NT and does not intersect with it. In this geometry and for a SWNT, it has been shown [7] that the energy loss suffered by the incident electron is proportional to the imaginary part of the polarisability and can be written as a function of $\operatorname{Im}(\varepsilon \perp)+\operatorname{Im}\left(-1 / \varepsilon_{/ /}\right)$ where $\varepsilon \perp$ and $\varepsilon_{/ /}$are respectively the in-plane and out-of-plane components of the dielectric tensor of a planar h-BN sheet. As the onset of the spectrum mainly reflects the contribution of $\operatorname{Im}(\varepsilon \perp)$, one can deduce the value of the optical gap, Fig. 2 (b). We found a homogeneous value around $5.8 \mathrm{eV}$ for all the tubes, independent of the number of walls or diameter of the tubes [1].

\section{References}

[1] R. Arenal, et al., Phys. Rev. Lett. 95, 127601 (2005).

[2] R.S. Lee et. al., Phys. Rev. B, 64, 121405(R) (2001).

[3] R. Arenal, O. Stephan, J.L. Cochon, A. Loiseau, in preparation.

[4] A. Gloter, A. Douiri, M. Tencé, C. Colliex, Ultramicroscopy 96, 385 (2003).

[5] L. Henrard, P. Lambin, J. Phys. B 29, 5127 (1996).

[6] C. Tarrio, S. Schnatterly, Phys. Rev. B 40, 7852 (1989).

[7] M. Kociak, et al., Phys. Rev. Lett. 87, 75501 (2001).
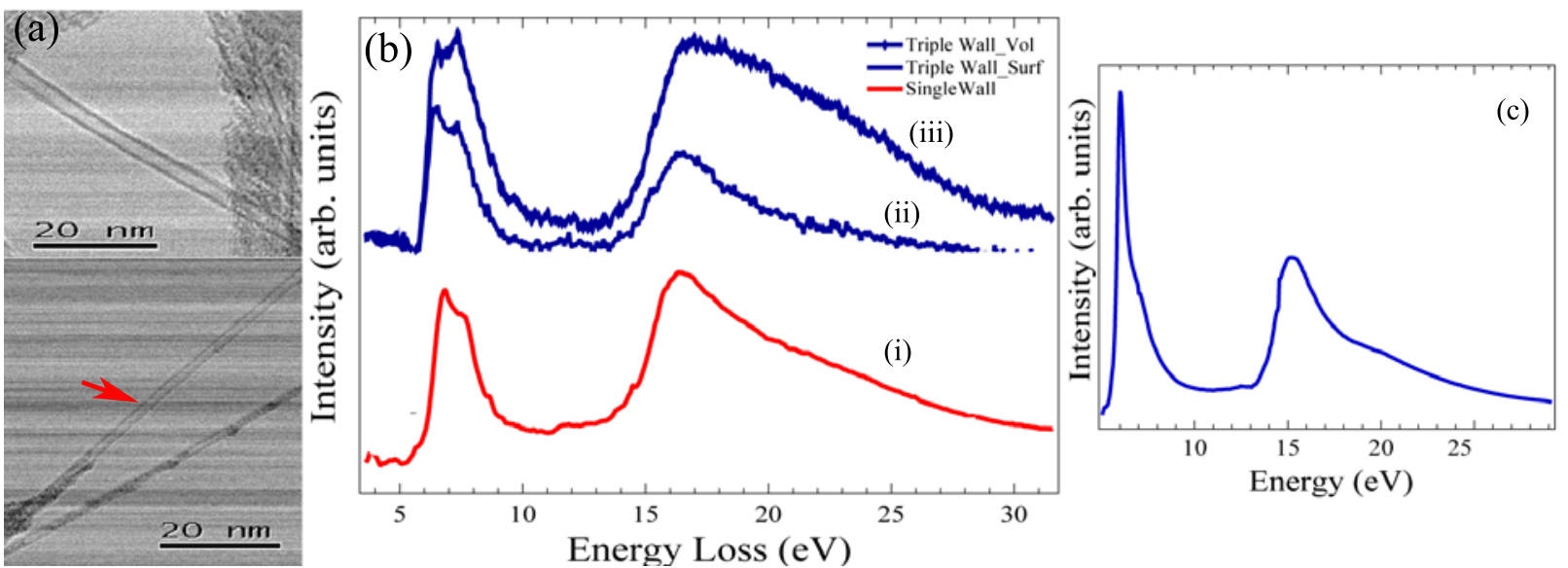

Figure 1. (a) Bright field images of a SW-BNNT (botton) and a TW-BNNT (top). (b) Experimental deconvoluted spectra for the SW (i) and for the TW ((ii) and (iii)). (c) $\operatorname{Im}(\varepsilon \perp)$. (b) and (c) see text.
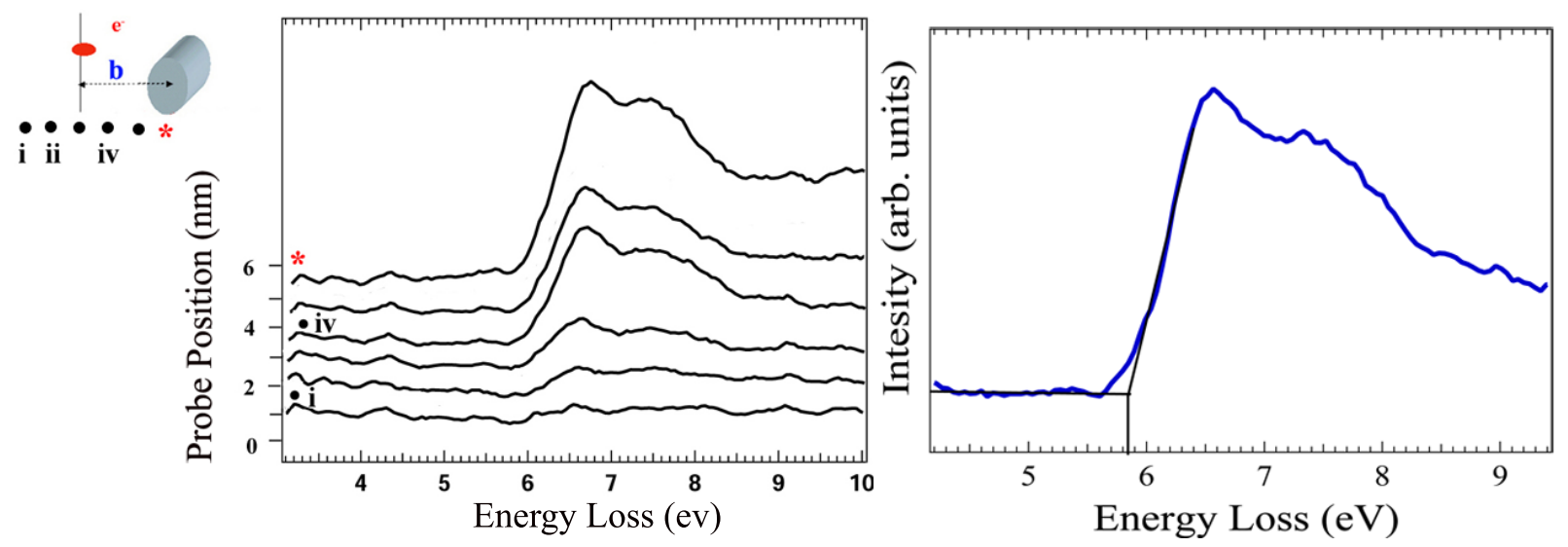

Figure 2. (a) Spectrum-line of a SW-BNNT (b) Measurement of the optical gap of a BNNT from one of the spectra extracted of Fig. 2 (a). 